\title{
INCLUSÃO DO EDUCADOR FÍSICO NA ATENÇÃO PRIMÁRIA À SAÚDE: REVISÃO INTEGRATIVA
}

\author{
INCLUDING THE PHYSICAL EDUCATOR IN PRIMARY HEALTH CARE:
} INTEGRATIVE REVIEW

INCLUSIÓN DEL EDUCADOR FÍSICO EN LA ATENCIÓN PRIMARIA DE SALUD: REVISIÓN INTEGRADORA

Jefferson Carlos Araujo Silva ${ }^{1}$

Kamila Santos da Silva ${ }^{2}$

Carlos Martins Neto ${ }^{3}$

Thalita Cristinny Araujo Silva ${ }^{4}$

Alexandre Soares de Campos ${ }^{5}$

Palavras-chave:

Educação Física; Atenção Primária à Saúde; Promoção da Saúde.

Keywords:

Physical Education; Primary Health Care; Health Promotion.

Palabras clave: Educación Física; Atención Primaria de Salud; Promoción de la Salud.

Submetido: 04/12/2017

Aprovado:

04/05/2018

Autor(a) para Correspondência: Jefferson Carlos Araujo Silva End: Centro Metropolitano, Conj. A, Lote 1 - CEP: 72220-275, Brasília-DF E-mail: jeffcasilva@gmail.com

\section{RESUMO}

O objetivo deste estudo foi analisar a inclusão do profissional de educação física (PEF) na atenção primária à saúde (APS). Trata-se de uma revisão integrativa de literatura, realizada com publicações do periodo entre 2008 e 2018 e buscas nas bases MedLine, SciELO, Lilacs, PubMed e Google Acadêmico, por meio dos seguintes descritores em ciências da saúde (DeCS): educação física, atenção primária à saúde, inclusão e promoção da saúde. A palavra-chave atenção básica foi considerada sinônimo do DeCS atenção primária à saúde. Foram identificados 67 artigos com a temática desta revisão; após a aplicação dos critérios de inclusão e exclusão, foram selecionados 10 artigos. Os estudos revelaram deficiência na formação do PEF em relação às temáticas da APS. Ressaltou-se o estímulo a estratégias como o Programa de Educação pelo Trabalho para a Saúde (PET-Saúde), que visam a suprir a carência de disciplinas específicas nos currículos de graduação em Educação Física, colocando o PEF em contato com a promoção da saúde desde esse nível de formação. Constatou-se que a atuação do PEF na APS deve abranger a prevenção de doenças crônico-degenerativas não transmissíveis e as ações de educação em saúde.

\footnotetext{
1. Fisioterapeuta. Aluno de Mestrado em Ciências da Reabilitação na Universidade de Brasília (UnB). Brasília (DF), Brasil. E-mail: jeffcasilva@gmail.com

2. Fisioterapeuta. Aluno de Mestrado em Ciência Biomédicas na Universidade Federal do Piauí (UFPI). Parnaíba (PI), Brasil. E-mail: kamilasantoskss@outlook.com

3. Fisioterapeuta. Residente em Terapia Intensiva no Hospital Universitário da Universidade Federal do Maranhão (HU-UFMA). São Luís (MA), Brasil. E-mail: carlosneto91@hotmail.com

4. Aluna de Graduação em Enfermagem no Centro Universitário Maurício de Nassau (Uninassau). Parnaíba (PI), Brasil. E-mail: cristinny.thalita@gmail.com

5. Educador Físico. Residente em Saúde Renal no HU-UFMA. São Luís (MA), Brasil. E-mail: alexandresoaresef@ gmail.com
} 


\section{ABSTRACT}

This study aimed to analyze the inclusion of the physical education professional (PEP) in primary health care $(P H C)$. This is an integrative literature review, conducted with publications from the period within 2008 and 2018 and searches in the databases MedLine, SciELO, LILACS, PubMed, and Google Academic, by means of the following descriptors in health sciences (DeCS): physical education, primary health care, inclusion, and health promotion. The keyword primary care was considered as a synonym of the DeCS primary health care. Sixty-seven articles were identified as having the theme of this review; after applying the inclusion and exclusion criteria, 10 articles were selected. The studies revealed a deficiency in the education of a PEP in relation to the themes of PHC. Emphasis was given to strategies such as the Program Education through Work for Health (PEW-Health), which aim to overcome the lack of specific subjects in undergraduate curricula in Physical Education, putting the PEP in contact with health promotion from this educational level onwards. It was found that the work of the PEP in PHC should cover the prevention of non-transmissible chronic-degenerative diseases and health education actions.

\section{RESUMEN}

Este estudio tuvo como objetivo analizar la inclusión del profesional de educación física (PEF) en la atención primaria de salud (APS). Se trata de una revisión integradora de la literatura, realizada con publicaciones del período entre 2008 y 2018 y búsquedas en las bases de datos MedLine, SciELO, LILACS, PubMed y Google Académico, mediante los siguientes descriptores en ciencias de la salud (DeCS): educación física, atención primaria de salud, inclusión y promoción de la salud. La palabra clave atención primaria se consideró como un sinónimo del DeCS atención primaria de salud. Se identificaron 67 artículos con el tema de esta revisión; después de aplicar los criterios de inclusión y exclusión, se seleccionaron 10 artículos. Los estudios revelaron una deficiencia en la formación del PEF con relación a los temas de la APS. Se resaltó el estímulo a estrategias como el Programa de Educación a través del Trabajo para la Salud (PET-Salud), que tienen como objetivo superar la falta de asignaturas específicas en los currículos de pregrado en Educación Física, poniendo al PEF en contacto con la promoción de la salud desde este nivel de formación. Se encontró que el trabajo del PEF en la APS debe cubrir la prevención de enfermedades crónico-degenerativas no transmisibles y las acciones de educación en salud.

\section{INTRODUÇÃO}

A reforma sanitária brasileira, de meados da década de 1980, culminou na criação do Sistema Único de Saúde (SUS) - instituído pela Constituição Federal de 1988 (CF/1988) - e levou à adoção de um novo modelo de atenção à saúde ${ }^{1,2}$. 0 paradigma vigente até então, denominado biomédico, já não se mostrava eficiente e envolvia altos custos, além de não atender à população brasileira como um todo ${ }^{3}$.

A CF/1988 dispõe em seu artigo 198, caput e incisos I a IIII:

Art. 198. As ações e serviços públicos de saúde integram uma rede regionalizada e hierarquizada e constituem um sistema único, organizado de acordo com as seguintes diretrizes:

I - descentralização, com direção única em cada esfera de governo;

II - atendimento integral, com prioridade para as atividades preventivas, sem prejuízo dos serviços assistenciais;

III - participação da comunidade ${ }^{4}$.

Assim, no Brasil, a saúde é um direito de todos e um dever do Estado ${ }^{2,4}$ e assume destaque a adoção de um modelo assistencial com vistas à promoção da saúde ${ }^{5}$.

Para sua efetivação, o SUS recorre a medidas ou estratégias, como o Programa de Agentes Comunitários de Saúde (Pacs) e a Estratégia Saúde da Família $(E S F)^{6}$, que buscam substituir o paradigma biomédico e reorganizar a atenção primária à saúde (APS) sobre novas bases, com a família como foco das políticas voltadas à promoção da saúde populacional?

A aprovação da Política Nacional da Atenção Básica (PNAB) pelo Ministério da Saúde (MS) reestruturou a $\mathrm{APS}^{8}$ e caracterizou a ESF como sua base de expansão, qualificação e consolidação, além de eixo estruturante do SUS. A ESF favorece a reorientação do processo de trabalho no sentido de aprofundar os princípios, as diretrizes e os fundamentos da APS 9 .

0 núcleo de apoio à saúde da família (Nasf) foi criado para aumentar a abrangência e a resolutividade da ESF ${ }^{10}$. 0 Nasf aumenta o escopo de ações da APS e a capacidade clínica das equipes de saúde, pois consiste em uma equipe com profissionais de diferentes áreas do conhecimento que atuam em conjunto com profissionais de equipes da ESF, 
compartilhando e apoiando as práticas em saúde nos territórios sob responsabilidade dessas equipes da $\mathrm{ESF}^{11}$.

0 profissional de educação física (PEF) faz parte da equipe do Nasf e atua por meio de práticas corporais e de atividade física sistematizada, seguindo a concepção de saúde coletiva em vez da individual ${ }^{12}$. 0 SUS indica que a prática regular de exercício físico, determinante para a saúde integral, deve ser estimulada e orientada por profissional capacitado ${ }^{13}$.

Sendo fundamental a inserção do PEF na APS com vistas à promoção à saúde e à prevenção e reabilitação de doenças - e por ser a ESF uma área relativamente nova de atuação do PEF, mostra-se importante reconhecer seu processo de trabalho e sua inserção nesse contexto. Diante do exposto, este artigo analisa a inclusão do PEF na APS.

\section{MATODOLOGIA}

Trata-se de uma revisão integrativa de literatura ${ }^{14}$, realizada com buscas nas bases MedLine, SciELO, Lilacs, PubMed e Google Acadêmico, por meio dos seguintes descritores em ciências da saúde (DeCS): educação física, atenção primária à saúde, inclusão e promoção da saúde. A palavra-chave atenção básica foi considerada sinônimo do DeCS atenção primária à saúde. Os termos de busca foram aplicados de modo individual e cruzado. 0s cruzamentos adotados foram: educação física e atenção básica (36 artigos); educação física e inclusão (12 artigos); e educação física e promoção da saúde (19 artigos). 0 período definidos para a inclusão de publicações foi entre 2008 e 2018, pois a inclusão do PEF na APS ocorreu mediante a Portaria GM n. 154, de 24 de janeiro de 2008, que criou os $\mathrm{Nasf}^{10}$.

Os critérios de inclusão adotados foram: a) artigos disponíveis na íntegra nas bases de dados em questão; b) publicações em português no período entre 2008 e 2018; e c) publicações sobre o processo de trabalho do PEF e sua inclusão na APS. Já os critérios de exclusão foram: a) publicações que não versavam sobre o processo de trabalho do PEF e sua inclusão na APS; b) resumos publicados em anais de congresso; e c) publicações duplicadas nas bases de dados em questão.

\section{RESULTADOS}

Foram identificados 67 artigos com a temática desta revisão integrativa de literatura. Após a aplicação dos critérios de inclusão e exclusão, foram selecionados 10 artigos - lidos na íntegra e caracterizados na Tabela 1.

Tabela 1 - Categorização dos artigos selecionados com base na aplicação dos critérios de inclusão e exclusão adotados.

\begin{tabular}{|c|c|c|c|c|}
\hline $\mathbf{N}$ & Autor(es)/ano & Título & Objetivo & $\begin{array}{l}\text { Tipo de } \\
\text { estudo }\end{array}$ \\
\hline 1 & $\begin{array}{l}\text { Anjos, Duarte } \\
\quad(2009)^{15}\end{array}$ & $\begin{array}{l}\text { A educação física e a } \\
\text { Estratégia Saúde da } \\
\text { Família: formação e } \\
\text { atuação profissional }\end{array}$ & $\begin{array}{l}\text { Analisar currículos de cursos de } \\
\text { graduação em Educação Física. }\end{array}$ & Descritivo \\
\hline 2 & $\begin{array}{l}\text { Santos et al. } \\
\qquad(2011)^{16}\end{array}$ & $\begin{array}{l}\text { Educação física e o } \\
\text { PET-Saúde: uma } \\
\text { estratégia } \\
\text { complementar na } \\
\text { formação para o SUS }\end{array}$ & $\begin{array}{l}\text { Salientar a importância do Programa de } \\
\text { Educação pelo Trabalho para a Saúde } \\
\text { (PET-Saúde) como formação } \\
\text { complementar do profissional de } \\
\text { educação física (PEF) para o Sistema } \\
\text { Único de Saúde (SUS). }\end{array}$ & $\begin{array}{l}\text { Qualitativo/ } \\
\text { descritivo }\end{array}$ \\
\hline 3 & $\begin{array}{l}\text { Santos, } \\
\text { Benedetti } \\
(2012)^{17}\end{array}$ & $\begin{array}{l}\text { Cenário de implantação } \\
\text { do núcleo de apoio à } \\
\text { saúde da família e a } \\
\text { inserção do profissional } \\
\text { de educação física }\end{array}$ & $\begin{array}{l}\text { Caracterizar a implantação do núcleo } \\
\text { de apoio à saúde da família (Nasf) e a } \\
\text { inserção do PEF no Brasil, tendo como } \\
\text { referência o mês de janeiro de } 2011 \text {. }\end{array}$ & $\begin{array}{l}\text { Descritivo/ } \\
\text { epidemiológico }\end{array}$ \\
\hline 4 & $\begin{array}{l}\text { Falci, Belisário } \\
\qquad(2013)^{18}\end{array}$ & $\begin{array}{c}\text { A inserção do } \\
\text { profissional de } \\
\text { educação física na } \\
\text { atenção primária à } \\
\text { saúde e os desafios em } \\
\text { sua formação }\end{array}$ & $\begin{array}{c}\text { Analisar a formação do PEF para sua } \\
\text { inserção na atenção primária à saúde } \\
\text { (APS). }\end{array}$ & $\begin{array}{l}\text { Qualitativo/ } \\
\text { exploratório }\end{array}$ \\
\hline
\end{tabular}




\begin{tabular}{|c|c|c|c|c|}
\hline $\mathrm{N}$ & Autor(es)/ano & Título & Objetivo & $\begin{array}{l}\text { Tipo de } \\
\text { estudo }\end{array}$ \\
\hline 5 & $\begin{array}{l}\text { Guarda et al. } \\
\qquad(2014)^{19}\end{array}$ & $\begin{array}{l}\text { A atividade física como } \\
\text { ferramenta de apoio às } \\
\text { ações da atenção } \\
\text { primária à saúde }\end{array}$ & $\begin{array}{c}\text { Apresentar as ações de atividades } \\
\text { físicas desenvolvidas pela equipe dos } \\
\text { Nasf em Igarassu (PE). }\end{array}$ & Descritivo \\
\hline 6 & $\begin{array}{l}\text { Lima et al. } \\
\qquad(2015)^{20}\end{array}$ & $\begin{array}{l}\text { Representações sociais } \\
\text { sobre o trabalho do } \\
\text { profissional de } \\
\text { educação física na } \\
\text { atenção primária à } \\
\text { saúde pelas equipes de } \\
\text { saúde da família }\end{array}$ & $\begin{array}{c}\text { Analisar as representações sociais } \\
\text { acerca do trabalho do PEF na APS pelos } \\
\text { profissionais das equipes da Estratégia } \\
\text { Saúde da Família (ESF). }\end{array}$ & Qualitativo \\
\hline 7 & $\begin{array}{l}\text { Silva, } \\
\text { Figueiredo } \\
\text { Júnior }(2015)^{21}\end{array}$ & $\begin{array}{l}\text { A atuação da educação } \\
\text { física no âmbito da } \\
\text { saúde pública: uma } \\
\text { revisão sistemática }\end{array}$ & $\begin{array}{c}\text { Mostrar a importância da inserção do } \\
\text { PEF nas ações desenvolvidas no âmbito } \\
\text { da saúde pública. }\end{array}$ & $\begin{array}{l}\text { Revisão } \\
\text { sistemática de } \\
\text { literatura }\end{array}$ \\
\hline 8 & $\begin{array}{l}\text { Carvalho, } \\
(2016)^{22}\end{array}$ & $\begin{array}{l}\text { Práticas corporais e } \\
\text { atividades físicas na } \\
\text { atenção básica do } \\
\text { sistema único de saúde: } \\
\text { ir além da prevenção } \\
\text { das doenças crônicas } \\
\text { não transmissíveis } \\
\text { (DCNT) é necessário }\end{array}$ & $\begin{array}{c}\text { Defender que as práticas corporais e } \\
\text { atividades físicas ofertadas na APS do } \\
\text { SUS não se resumam a atividades de } \\
\text { prevenção das DCNT. }\end{array}$ & $\begin{array}{l}\text { Revisão de } \\
\text { literatura não } \\
\text { sistematizada }\end{array}$ \\
\hline 9 & $\begin{array}{l}\text { Ferreira et al. } \\
\qquad(2016)^{23}\end{array}$ & $\begin{array}{c}\text { A inserção do } \\
\text { profissional de } \\
\text { educação física nos } \\
\text { núcleos de apoio a } \\
\text { saúde da família: visão } \\
\text { dos profissionais }\end{array}$ & $\begin{array}{c}\text { Analisar a percepção do PEF inserido } \\
\text { no Nasf sobre as funções e a } \\
\text { efetividade da intervenção } \\
\text { multiprofissional nas equipes em São } \\
\text { Paulo (SP). }\end{array}$ & $\begin{array}{l}\text { Qualitativo/ } \\
\text { descritivo }\end{array}$ \\
\hline 10 & $\begin{array}{l}\text { Santos et al. } \\
\qquad(2016)^{24}\end{array}$ & $\begin{array}{c}\text { Representações sociais } \\
\text { dos usuários do Sistema } \\
\text { Único de Saúde do } \\
\text { Brasil acerca das } \\
\text { práticas corporais/ } \\
\text { atividades físicas na } \\
\text { atenção primária à } \\
\text { saúde }\end{array}$ & $\begin{array}{c}\text { Conhecer as representações sociais dos } \\
\text { usuários do SUS acerca das práticas } \\
\text { corporais e das atividades físicas } \\
\text { realizadas na APS. }\end{array}$ & $\begin{array}{l}\text { Qualitativo/ } \\
\text { descritivo }\end{array}$ \\
\hline
\end{tabular}

Fonte: Elaborada pelos autores.

A Tabela 2 apresenta a síntese dos estudos selecionados em relação às características de sua amostra, aos seus principais resultados e à sua conclusão.

Tabela 2 - Síntese dos artigos selecionados com base na aplicação dos critérios de inclusão e exclusão adotados.

\begin{tabular}{|c|c|c|c|c|}
\hline N & Autor(es)/ano & Amostra & Principais resultados & Conclusão \\
\hline 1 & $\begin{array}{c}\text { Anjos, Duarte } \\
(2009)^{15}\end{array}$ & $\begin{array}{c}\text { Grade curricular } \\
\text { de } 4 \text { faculdades } \\
\text { de Educação } \\
\text { Física. }\end{array}$ & $\begin{array}{l}\text { 0s cursos apresentam } \\
\text { atividades direcionadas ao } \\
\text { campo da saúde, mas não } \\
\text { de forma específica. }\end{array}$ & $\begin{array}{c}\text { Há carência de informações } \\
\text { sobre os serviços públicos de } \\
\text { saúde e a atuação do } \\
\text { profissional de educação física } \\
\text { (PEF) em sua formação. Sugere- } \\
\text { se que repensem e incluam } \\
\text { formas de capacitação, para que } \\
\text { a Educação Física assuma com } \\
\text { propriedade seu novo campo de } \\
\text { atuação. }\end{array}$ \\
\hline
\end{tabular}




\begin{tabular}{|c|c|c|c|c|}
\hline $\mathrm{N}$ & Autor(es)/ano & Amostra & Principais resultados & Conclusão \\
\hline 2 & $\begin{array}{l}\text { Santos et al. } \\
\quad(2011)^{16}\end{array}$ & $\begin{array}{c}\text { Diálogos } \\
\text { registrados nos } \\
\text { diários de campo } \\
\text { do Programa de } \\
\text { Educação pelo } \\
\text { Trabalho para a } \\
\text { Saúde (PET- } \\
\text { Saúde). } \\
\text { Acadêmicos, } \\
\text { preceptores, } \\
\text { tutores e } \\
\text { profissionais do } \\
\text { campo de atuação } \\
\text { que registravam } \\
\text { suas atividades } \\
\text { nos diários. }\end{array}$ & $\begin{array}{l}\text { A maioria dos currículos dos } \\
\text { cursos de Educação Física } \\
\text { não trabalha a inserção } \\
\text { desse profissional na } \\
\text { atenção primária à saúde } \\
\text { (APS). O estudante de } \\
\text { Educação Física deve buscar } \\
\text { referências que apoiem essa } \\
\text { prática e inserir-se em } \\
\text { campo de estágio para que } \\
\text { haja maior interação no } \\
\text { processo de serviço na } \\
\text { saúde coletiva. }\end{array}$ & $\begin{array}{l}\text { 0 PET-Saúde realiza a } \\
\text { integração ensino-serviço- } \\
\text { comunidade e capacita o } \\
\text { estudante universitário para a } \\
\text { vivência no âmbito da saúde } \\
\text { coletiva, de modo } \\
\text { interdisciplinar. }\end{array}$ \\
\hline 3 & $\begin{array}{c}\text { Santos, } \\
\text { Benedetti } \\
(2012)^{17}\end{array}$ & $\begin{array}{c}\text { Dados } \\
\text { secundários do } \\
\text { Departamento de } \\
\text { Informática do } \\
\text { Sistema Único de } \\
\text { Saúde (DataSUS), } \\
\text { em específico no } \\
\text { Cadastro Nacional } \\
\text { dos } \\
\text { Estabelecimentos } \\
\text { de Saúde (CNES). }\end{array}$ & $\begin{array}{l}\text { A Educação Física está } \\
\text { entre as } 5 \text { profissões mais } \\
\text { recrutadas para atuação no } \\
\text { núcleo de apoio à saúde da } \\
\text { família (Nasf). } 0 \\
\text { coeficiente de PEF por } \\
\text { população coberta pela } \\
\text { Estratégia Saúde da Família } \\
\text { (ESF) é inferior a } 1 \\
\text { profissional por } 100 \text { mil } \\
\text { habitantes. A estimativa foi } \\
\text { de } 0,25 \text { ou menos } \\
\text { profissionais por } 100 \text { mil } \\
\text { habitantes cobertos pela } \\
\text { ESF. }\end{array}$ & $\begin{array}{l}\text { Nota-se a necessidade de } \\
\text { investimento político e } \\
\text { acadêmico para definição de } \\
\text { estratégias coletivas que } \\
\text { contribuam para a consolidação } \\
\text { do Nasf e a integração do PEF } \\
\text { na rede de assistência pública } \\
\text { de saúde. }\end{array}$ \\
\hline 4 & $\begin{array}{c}\text { Falci, Belisário } \\
(2013)^{18}\end{array}$ & $\begin{array}{l}\text { Dois grupos, de } 7 \\
\text { e } 8 \text { pessoas, } \\
\text { compostos por } \\
\text { PEF que cursam } \\
\text { especialização em } \\
\text { APS, com } \\
\text { experiência na } \\
\text { APS e próximos } \\
\text { de finalizar esse } \\
\text { curso. }\end{array}$ & $\begin{array}{c}\text { A inserção do PEF na APS } \\
\text { foi vista como ampliação do } \\
\text { mercado de trabalho e } \\
\text { deve-se ao crescimento das } \\
\text { doenças crônicas não } \\
\text { transmissíveis (DCNT) e ao } \\
\text { incentivo às políticas } \\
\text { públicas de saúde. } \\
\text { Entretanto, ressaltam-se } \\
\text { déficits na formação para a } \\
\text { inserção do profissional na } \\
\text { APS. }\end{array}$ & $\begin{array}{l}\text { A inserção do PEF na APS reflete } \\
\text { de modo positivo nos serviços e } \\
\text { na comunidade. Deve-se atentar } \\
\text { para a formação, a fim de } \\
\text { promover maior inserção do } \\
\text { estudante universitário nesse } \\
\text { novo âmbito de trabalho. }\end{array}$ \\
\hline 5 & $\begin{array}{l}\text { Guarda et al. } \\
\qquad(2014)^{19}\end{array}$ & $\begin{array}{l}\text { Registro das } \\
\text { ações } \\
\text { desenvolvidas } \\
\text { pelo PEF } \\
\text { integrante do } \\
\text { Nasf. }\end{array}$ & $\begin{array}{c}\text { As atividades são } \\
\text { desenvolvidas por } 2 \\
\text { profissionais. São } \\
\text { executadas aulas de } \\
\text { ginástica, atividades em } \\
\text { grupos, danças de salão e } \\
\text { apoio às atividades de } \\
\text { promoção à saúde } \\
\text { desenvolvidas pelas equipes } \\
\text { da ESF. }\end{array}$ & $\begin{array}{c}\text { O Nasf atua promovendo } \\
\text { integralidade, participação } \\
\text { social e intersetorialidade. Visa } \\
\text { a desenvolver ações no } \\
\text { território que capacitem os } \\
\text { sujeitos a intervir em aspectos } \\
\text { individuais e coletivos da } \\
\text { saúde. }\end{array}$ \\
\hline
\end{tabular}




\begin{tabular}{|c|c|c|c|c|}
\hline $\mathbf{N}$ & Autor(es)/ano & Amostra & Principais resultados & Conclusão \\
\hline 6 & $\begin{array}{l}\text { Lima et al. } \\
\qquad(2015)^{20}\end{array}$ & $\begin{array}{l}12 \text { profissionais } \\
\text { de saúde que } \\
\text { atuam na ESF e } \\
\text { que contam com } \\
\text { o trabalho do PEF } \\
\text { no Nasf. }\end{array}$ & $\begin{array}{l}0 \text { PEF foi reconhecido como } \\
\text { atuante em grupos } \\
\text { específicos. Alguns } \\
\text { profissionais da saúde } \\
\text { afirmaram desconhecer o } \\
\text { trabalho do PEF. Houve } \\
\text { relação entre atividade } \\
\text { física/prática corporal e } \\
\text { prevenção de doenças e/ou } \\
\text { sedentarismo. }\end{array}$ & $\begin{array}{l}\text { Há boa visibilidade das } \\
\text { atividades desenvolvidas pelo } \\
\text { PEF, mesmo que alguns } \\
\text { profissionais da saúde } \\
\text { desconheçam seu trabalho. } \\
\text { Salienta-se o déficit do curso } \\
\text { de graduação no preparo para } \\
\text { esse campo de trabalho. }\end{array}$ \\
\hline 7 & $\begin{array}{c}\text { Silva, Figueiredo } \\
\text { Júnior }(2015)^{21}\end{array}$ & $\begin{array}{l}8 \text { artigos que } \\
\text { discorrem sobre a } \\
\text { atuação do PEF } \\
\text { no âmbito da } \\
\text { saúde pública. }\end{array}$ & $\begin{array}{l}\text { 0 PEF atuante na saúde } \\
\text { pública pode contribuir } \\
\text { substancialmente para } \\
\text { melhorar a qualidade de } \\
\text { vida da população. }\end{array}$ & $\begin{array}{l}\text { A inserção do PEF na APS se } \\
\text { mostrou necessária e seu } \\
\text { trabalho sempre absorve um } \\
\text { caráter multidisciplinar. Há } \\
\text { déficits na formação desse } \\
\text { profissional em relação a esse } \\
\text { campo de atuação. }\end{array}$ \\
\hline 8 & $\begin{array}{l}\text { Carvalho, } \\
(2016)^{22}\end{array}$ & $\begin{array}{c}\text { Informações } \\
\text { coletadas em } \\
\text { periódicos, livros } \\
\text { e documentos } \\
\text { oficiais. }\end{array}$ & $\begin{array}{c}\text { A atividade física } \\
\text { proporciona, além dos } \\
\text { benefícios biológicos } \\
\text { individuais, a inserção em } \\
\text { grupos com objetivos em } \\
\text { comum. }\end{array}$ & $\begin{array}{l}\text { As práticas corporais e as } \\
\text { atividades físicas na APS devem } \\
\text { ampliar e garantir a } \\
\text { integralidade do cuidado e não } \\
\text { só os benefícios } \\
\text { biopsicossociais. }\end{array}$ \\
\hline 9 & $\begin{array}{l}\text { Ferreira et al. } \\
\qquad(2016)^{23}\end{array}$ & $\begin{array}{l}8 \text { PEF com } \\
\text { experiência de } \\
\text { trabalho no Nasf. }\end{array}$ & $\begin{array}{l}\text { Os profissionais tinham } \\
\text { conhecimento sobre o } \\
\text { processo de trabalho no } \\
\text { Nasf e afirmaram que o PEF } \\
\text { é responsável pela } \\
\text { realização de atividades } \\
\text { física em grupo, avaliação } \\
\text { física, orientação de } \\
\text { hábitos saudáveis, auxílio } \\
\text { na saúde mental etc. }\end{array}$ & $\begin{array}{l}\text { 0s PEF detêm conhecimento } \\
\text { sobre seu papel de atuação no } \\
\text { Nasf, porém, com limitações que } \\
\text { não devem ser ignoradas. }\end{array}$ \\
\hline 10 & $\begin{array}{l}\text { Santos et al. } \\
\qquad(2016)^{24}\end{array}$ & $\begin{array}{l}5 \text { praticantes e } 5 \\
\text { não praticantes } \\
\text { de atividade } \\
\text { física. }\end{array}$ & $\begin{array}{c}\text { 0s indivíduos que } \\
\text { praticavam atividade física } \\
\text { buscavam melhorar suas } \\
\text { condições de saúde. 0s não } \\
\text { praticantes alegaram } \\
\text { desconhecimento das } \\
\text { atividades promovidas pelo } \\
\text { Nasf. }\end{array}$ & $\begin{array}{c}\text { A comunidade, ao participar da } \\
\text { construção de meios para } \\
\text { melhoria da saúde, apropria-se } \\
\text { do empoderamento. O PEF } \\
\text { apresenta formação voltada ao } \\
\text { Sistema Único de Saúde (SUS) } \\
\text { por meio da residência } \\
\text { multiprofissional. }\end{array}$ \\
\hline
\end{tabular}

Fonte: Elaborada pelos autores.

\section{DISCUSSÃO}

A inclusão do PEF na APS visa a coordenar, planejar e realizar treinamentos especializados, com ênfase nas práticas corporais e nas atividades físicas sistematizadas. 0 PEF tem um trabalho voltado à promoção da saúde, prevenindo e tratando, de modo não farmacológico, as doenças crônicas não transmissíveis $(D C N T)^{15}$. A atuação do PEF no Nasf visa a oferecer suporte especializado para que a população realize atividade física sistematizada sob supervisão de profissional capacitado ${ }^{16}$.
Um estudo ${ }^{15}$, ao analisar o currículo dos cursos de graduação em Educação Física, observou que nenhuma das instituições em questão apresentava direcionamento dos cursos à APS. Quando comparados os cursos de bacharelado aos de licenciatura, observase nos primeiros melhor preparo para trabalhar na APS, por possuírem maior número de disciplinas relacionadas ao tema. Esse fato evidencia carência desde o processo de formação do PEF, que não é voltado à atuação na APS.

0 Programa de Educação pelo Trabalho para a Saúde (PET-Saúde) foi criado para suprir tais carências 
dos cursos de graduação no setor saúde, visando a fomentar a formação, em nível de graduação, voltada à ESF por meio de educação tutorial ${ }^{17}$. Uma pesquisa ${ }^{16}$ procurou analisar a importância do PET-Saúde como parte complementar da formação do PEF para sua inclusão no SUS e na APS. Os autores concluíram que as experiências vivenciadas no PET-Saúde serviram de base para a atuação na APS e muniram o PEF de conhecimentos adequados acerca do SUS.

A criação do Nasf ${ }^{10}$ efetivou o PEF como profissional atuante na APS. Uma pesquisa ${ }^{17}$ realizada com dados secundários do SUS e do cadastro das equipes do Nasf caracterizou a implantação deste e a inserção do PEF nele e observou-se que tal implantação não havia ocorrido na maioria dos municípios brasileiros na época do estudo. A região Sudeste apresentou o maior número de equipes, ao passo que a região Norte apresentou o menor número de equipes. Ao analisar a inserção do PEF na APS, os autores observaram que ele foi o quinto profissional mais recrutado para os Nasf. Quanto à inserção do PEF no Nasf, verificou-se que $49,2 \%$ das equipes possuíam profissional em seu quadro, porém, os valores diferenciam entre os vários estados do país, apresentando proporções desiguais.

Pesquisadores selecionaram alunos de um curso de especialização em APS em Minas Gerais e avaliaram o processo de formação do PEF e sua inserção na APS. Concluiu-se que a inserção do PEF no Nasf proporcionou maior interdisciplinaridade na promoção à saúde, qualificando esse profissional da saúde, ao aumentar o escopo de suas ações e seu mercado de trabalho. Dentre as dificuldades identificadas, a formação do PEF foi considerada insuficiente para sua atuação na APS - pois ainda é focada em treinamento esportivo ${ }^{18}$.

A inserção do PEF no SUS é pouco explorada diante da significativa contribuição que pode proporcionar atuando na prevenção e no controle das DCNT, com base na prática regular de exercício físico planejando e na execução de atividades voltadas à promoção à saúde, com vistas ao caráter educativo. 0 PEF também pode orientar a construção de ciclovias e pistas de caminhada ${ }^{18}$. A prática regular de exercício físico em grupos especiais ${ }^{19}$, como os idosos, os hipertensos, os diabéticos, as gestantes e os portadores de transtornos mentais, entre outros, é um meio de intercâmbio de saberes com outras áreas da saúde, incorporando o PEF ao caráter interdisciplinar que a ESF propõe $\mathrm{e}^{19}$.

Alguns estudos apontaram que membros da equipe multidisciplinar não apresentam conhecimento

\section{...a formação dos grupos de atividade física promovem 0 fortalecimento das relações sociais...}

adequado sobre o trabalho do PEF na APS ${ }^{18,19}$. Uma pesquisa realizada em Limoeiro do Norte (CE ${ }^{20}$ analisou as representações sociais acerca do trabalho do PEF na APS por parte dos profissionais que compõem as equipes da ESF do município. 0s profissionais da ESF declararam que a atuação do PEF se mostra importante no que tange aos grupos de idosos e gestantes, no desenvolvimento de atividades físicas e práticas corporais. Constatou-se reconhecimento em relação ao trabalho interprofissional, por meio de atividades realizadas em conjunto com outros membros da equipe de saúde. Algumas dificuldades foram enumeradas, como a não valorização da categoria nas políticas públicas, o desconhecimento do trabalho do PEF por parte de alguns membros da equipe de saúde e a não capacitação, enquanto aluno de graduação, para desenvolver atividades na APS e inserir-se no Nasf.

Uma revisão sistemática de literatura ${ }^{21}$ destacou que o PEF não atua somente na promoção da prática de atividade física sistematizada, mas também na elaboração de projetos voltados ao caráter educativo da população. Outro ponto recorrente nessa revisão foi a interdisciplinaridade, visto que, inserido em uma equipe, participa conjuntamente na tomada de decisões para a prevenção, o tratamento e a reabilitação de usuários da ESF. 0 entendimento de que o exercício físico promove melhora da qualidade de vida - não somente visando à perda de peso, mas também à redução dos riscos de desenvolver DCNT e à prevenção do quadro de osteoporose, bem como dos efeitos adversos do processo natural de envelhecimento ${ }^{15-17}$ - levou a maior adoção da prática regular de exercício físico e maior visibilidade do PEF.

Uma pesquisa ${ }^{22}$ indicou que a atividade física estruturada ofertada no âmbito da APS não é exclusivamente voltada à prevenção de DCNT, mas também para alcançar objetivos socioafetivos, biológicos e cognitivos. A convivência e a formação dos grupos de atividade física promovem 0 fortalecimento das relações sociais, a prática de 
atividade física faz parte de um contexto maior, onde seu objetivo é a garantia da integralidade do cuidado, trazendo não só benefícios biológicos, mas também aumento do círculo social de convivência ${ }^{23}$.

Um estudo de abordagem qualitativa observou certo distanciamento do PEF em relação aos outros profissionais do Nasf e a principal função atribuída ao PEF foi a promoção da saúde, porém, destacou-se em alguns discursos o trabalho com a gestão, as políticas de saúde e o diagnóstico, entre outros aspectos ${ }^{23}$. Outro estudo qualitativo, realizado no norte do Paraná, constatou que houve resistência inicial por parte de alguns profissionais da ESF quanto à inclusão do PEF na APS. Os autores concluíram que a inserção do PEF na APS carece de maior preparo nos cursos de graduação, pois ainda é bastante voltada à prática esportiva generalista ${ }^{24-31}$.

Um estudo realizado em João Pessoa $(P B)^{32}$ identificou que todos os PEF possuíam diploma de Licenciatura Plena em Educação Física, visto que sua formação precedia a divisão dos cursos em bacharelado e licenciatura, e a maioria estava inserida em atividades de educação permanente. Um trabalho ${ }^{20}$ desenvolvido em Sobral (CE) analisou a visão do usuário acerca da inserção do PEF na APS; os usuários expressaram em seus discursos que o PEF contribui para a manutenção da saúde, porém, destacou-se a deficiência da divulgação das atividades promovidas pelo Nasf.

\section{CONCLUSÃO}

Este artigo evidenciou a importância do PEF na APS por meio dos Nasf e sua atuação não deve resumir-se à prevenção das DCNT; também se deve buscar a promoção da saúde por meio de palestras educativas.

0 PEF atua com maior destaque em grupos específicos, como os idosos ou as gestantes, mas não deve restringir-se a tais grupos. Destacou-se carência na formação do PEF quanto ao seu direcionamento para a atuação na APS; para superar esse desafio foram criadas medidas como o PET-Saúde e a residência multiprofissional em saúde.

Em suma, pode-se dizer que a atuação do PEF é considerada importante e complementar tanto por outros membros da equipe como pelos usuários.

\section{CONTRIBUIÇÃO DOS AUTORES}

Jefferson Carlos Araujo Silva contribuiu com o delineamento e a realização da pesquisa e a estruturação e redação do manuscrito. Kamila Santos da Silva, Carlos Martins Neto e Thalita Cristinny Araujo Silva contribuíram com a redação do manuscrito. Alexandre Soares de Campos contribuiu com a revisão crítica do manuscrito.

\section{REFERÊNCIAS}

1. Brasil. Histórico: do sanitarismo à municipalização. Brasília (DF): Ministério da Saúde; 2013.

2. Santos NR. Desenvolvimento do SUS, rumos estratégicos e estratégias para visualização dos rumos. Ciênc Saúde Colet [serial on the internet]. 2007 [cited 2018 May 7];12(2):42935. Available from: http://www.scielo.br/pdf/csc/v12n2/ a19v12n2.pdf

3. Oliveira DC, Sá CP, Gomes AMT, Ramos RS, Pereira NA, Santos WCR. A política pública de saúde brasileira: representação e memória social dos profissionais. Cad Saúde Pública. 2008;24(1):197-206.

4. Brasil. Constituição 1988. Constituição da República Federativa do Brasil. Brasília (DF): Senado; 1988.

5. Paim JS, Teixeira CF. Configuração institucional e gestão do Sistema Único de Saúde: problemas e desafios. Ciênc Saúde Colet [serial on the internet]. 2007 [cited 2018 May 7];12(Suppl):1819-29. Available from: http://www.scielo. $\mathrm{br} / \mathrm{pdf} / \mathrm{csc} / \mathrm{v} 12 \mathrm{~s} 0 / 05 . \mathrm{pdf}$

6. Brasil. Portal da Saúde [homepage on the internet]. 2017 [cited 2018 May 7]. Available from: http://u.saude.gov.br/ index.php/cidadao/acoes-e-programas

7. Ronzani TM, Silva CM. O Programa Saúde da Família segundo profissionais de saúde, gestores e usuários. Ciênc Saúde Colet [serial on the internet]. 2008 [cited 2018 May 7];13(1): 23-34. Available from: http://www.scielo.br/pdf/ csc/v13n1/06.pdf

8. Brasil. Política Nacional de Atenção Básica. Brasília (DF): Ministério da Saúde; 2012.

9. Piovesan LR, Schimith MD, Simon BS, Budó MLD, Weiller TH, Brêtas ACP. Promoção da saúde na perspectiva de enfermeiros de atenção básica. Rev Enferm UERJ [serial on the internet]. 2016 [cited 2018 May 7];24(3):e5816. Available from: http://www.e-publicacoes.uerj.br/index. php/enfermagemuerj/article/view/5816/19396

10. Brasil. Portaria GM n. 154, de 24 de janeiro de 2008. Cria Núcleos de Apoio à Saúde da Família - NASF. Diário Oficial da União [serial on the internet], Brasília (2008 Jan 25 [cited 2018 May 7]); Sec 1. Available from: http://189.28.128.100/dab/docs/legislacao/ portaria154_24_01_08.pdf 
11. Ribeiro MDA, Bezerra EMA, Costa MS, Branco CEC, Araújo Neto JD, Moreira AKF, et al. Avaliação da atuação do núcleo de apoio à saúde da família. Rev Bras Promoç Saúde [serial on the internet]. 2014 [cited 2018 May 7];27(2):224-31. Available from: http://periodicos.unifor.br/RBPS/article/ view/2426/pdf

12. Brasil. Núcleo de Apoio à Saúde da Família. Brasília (DF): Ministério da Saúde; 2014.

13. Fraga AB, Carvalho YM, Gomes IM. Políticas de formação em educação física e saúde coletiva. Trab Educ Saúde [serial on the internet]. 2012 [cited 2018 May 7];10(3):367-86. Available from: http://www.scielo.br/pdf/tes/v10n3/ a02v10n3.pdf

14. Botelho LLR, Cunha CCA, Macedo M. 0 método da revisão integrativa nos estudos organizacionais. Gestão e Sociedade [serial on the internet]. 2011 [cited 2018 May 7];5(11):12136. Available from: https://www.gestaoesociedade.org/ gestaoesociedade/article/view/1220/906

15. Anjos TC, Duarte ACGO. A educação física e a Estratégia Saúde da Família: formação e atuação profissional. Physis (Rio J). 2009;19(4):1127-44.

16. Santos ALB, Maia Neto JP, Souza Neto FCV, Oliveira BN, Rui EM, Sá MEG. Educação física e o PET-Saúde: uma estratégia complementar na formação para o SUS. Sanare (Sobral, Online) [serial on the internet]. 2011 [cited 2018 May 7];10(2):75-8. Available from: https://sanare. emnuvens.com.br/sanare/article/view/259/232

17. Santos SFS, Benedetti TRB. Cenário de implantação do núcleo de apoio à saúde da família e a inserção do profissional de educação física. Rev Bras Ativ Fís Saúde. 2012;17(3):188-94.

18. Falci DM, Belisário SA. A inserção do profissional de educação física na atenção primária à saúde e os desafios em sua formação. Interface Comun Saúde Educ [serial on the internet]. 2013 [cited 2018 May 7];17(47):885-99. Available from: http://www.scielo.br/pdf/icse/v17n47/aop3913.pdf

19. Guarda FRB, Silva RN, Silva SM, Santana PR. A atividade física como ferramenta de apoio às ações da atenção primária à saúde. Rev Bras Ativ Fís Saúde [serial on the internet]. 2014 [cited 2018 May 7];19(2):265-270. Available from: http://rbafs.emnuvens.com.br/RBAFS/article/ view/2756/pdf168

20. Lima LJR, Oliveira BN, Oliveira BN. Representações sociais sobre o trabalho do profissional de educação física na atenção primária à saúde pelas equipes de saúde da família. Educação Física em Revista [serial on the internet]. 2015 [cited 2018 May 7];9(2):12-22. Available from: https://portalrevistas.ucb.br/index.php/efr/article/ view/6575/4724

21. Silva JM, Figueiredo Júnior JM. A atuação da educação física no âmbito da saúde pública: uma revisão sistemática. Revista Brasileira de Educação e Saúde [serial on the internet]. 2015 [cited 2018 May 7];5(1):75-86. Available from: http://www.gvaa.com.br/revista/index.php/REBES/ article/view/3208/2703

22. Carvalho FFB. Práticas corporais e atividades físicas na atenção básica do Sistema Único de Saúde: ir além da prevenção das doenças crônicas não transmissiveis é necessário. Movimento (Porto Alegre) [serial on the internet]. 2016 [cited 2018 May 7];22(2):647-58. Available from: $\quad$ http://www.seer.ufrgs.br/index.php/Movimento/ article/view/58174/37391

23. Ferreira TB, Cipolotti MD, Marques BG, Miranda MLJ. A inserção do profissional de educação física nos núcleos de apoio a saúde da família: visão dos profissionais. Rev Bras Ativ Fís Saúde [serial on the internet]. 2016 [cited 2018 May 7];21(3):228-36. Available from: http://rbafs. emnuvens.com.br/RBAFS/article/view/5703/6025

24. Santos ALB, Aguiar JB, Gurgel LA, Silveira MAA, Catunda KC, Souza Neto FCV. Representações sociais dos usuários do Sistema Único de Saúde do Brasil acerca das práticas corporais/atividades físicas na atenção primária à saúde. $V$ Congresso Ibero-Americano em Investigação Qualitativa. Atas [document on the internet]. Porto: [s.n]; 2016 [cited 2018 May 7]. Available from: http://proceedings.ciaiq.org/ index.php/ciaiq2016/article/view/901/885

25. Corrêa LQ, Valério MP, Teixeira A0, Guerreiro LF, Silveira DF, Machado PT, et al. A atuação da educação física nas residências multiprofissionais em saúde. Rev Bras Promoç Saúde [serial on the internet]. 2014 [cited 2018 May 7];27(3):428-33. Available from: http://periodicos.unifor. br/RBPS/article/view/2863/pdf

26. Lopes ACS, Ferreira AD, Mendonça RD, Dias MAS, Rodrigues RCLC, Santos LC. Estratégia de promoção à saúde: programa academia da cidade de Belo Horizonte. Rev Bras Ativ Fís Saúde [serial on the internet]. 2016 [cited 2018 May 7];21(4):379-84. Available from: http://rbafs. emnuvens.com.br/RBAFS/article/view/7582/pdf

27. Souza FL, Silva MMS, Dias MSA, Vasconcelos MI0, Aguiar $C P$, Evangelista $A R$, et al. Formação dos monitores do PRÓPET-SAÚDE a partir das necessidades de aprendizagem vivenciadas no PSE. Tempus (Brasília) [serial on the internet]. 2015 [cited 2018 May 7];9(1):79-89. Available from: http://www.tempusactas.unb.br/index.php/tempus/ article/view/1693/1400

28. Guarda FB, Silva RN, Araújo Júnior JL, Santana PR, Santos Neto PM. Incorporação e contribuições dos profissionais de educação física ao Sistema Único de Saúde do Brasil. Tempus (Brasília) [serial on the internet]. 2014 [cited 2018 May 7];8(3):185-96. Available from: http:// tempusactas.unb.br/index.php/tempus/article/view/1573

29. Vieira ELS. Investigando a atuação dos profissionais de educação física das equipes do núcleo de apoio à saúde da família do município de Natal-RN. Natal: Universidade Federal do Rio Grande do Norte; 2016. 
30. Oliveira FA, Pirajá WC, Silva AP, Primo CPF. Benefícios da prática de atividade física sistematizada no lazer de idosos: algumas considerações. Licere [serial on the internet]. 2015 [cited 2018 May 7];18(2):262-304. Available from: $\quad$ https://seer.ufmg.br/index.php/licere/article/ view/1052/761

31. Souza SC, Loch MR. Intervenção do profissional de educação física nos núcleos de apoio à saúde da família em municípios do norte do Paraná. Rev Bras Ativ Fís Saúde [serial on the internet]. 2011 [cited 2018 May 7];16(1):510. Available from: http://rbafs.emnuvens.com.br/RBAFS/ article/view/549/547

32. Rodrigues JD, Ferreira DKS, Junior JCF, Caminha I0, Florindo AA, Loch MR. Perfil e atuação do profissional de educação física nos núcleos de apoio à saúde da família na região metropolitana de João Pessoa-PB. Rev Bras Ativ Fís Saúde [serial on the internet]. 2015 [cited 2018 May 7];20(4):352-65. Available from: http://rbafs.emnuvens. com.br/RBAFS/article/view/5098/4970

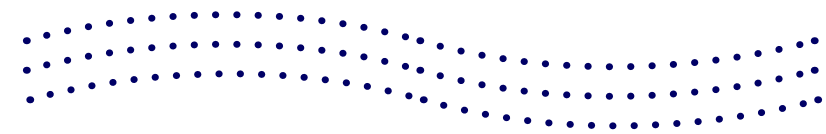

$\ldots \ldots \ldots \cdots \cdots$

$\ldots \ldots \ldots \ldots \ldots \ldots \ldots \ldots \ldots \ldots$ $\ldots \ldots \ldots \ldots \ldots \ldots \ldots \ldots$

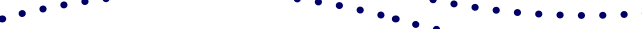
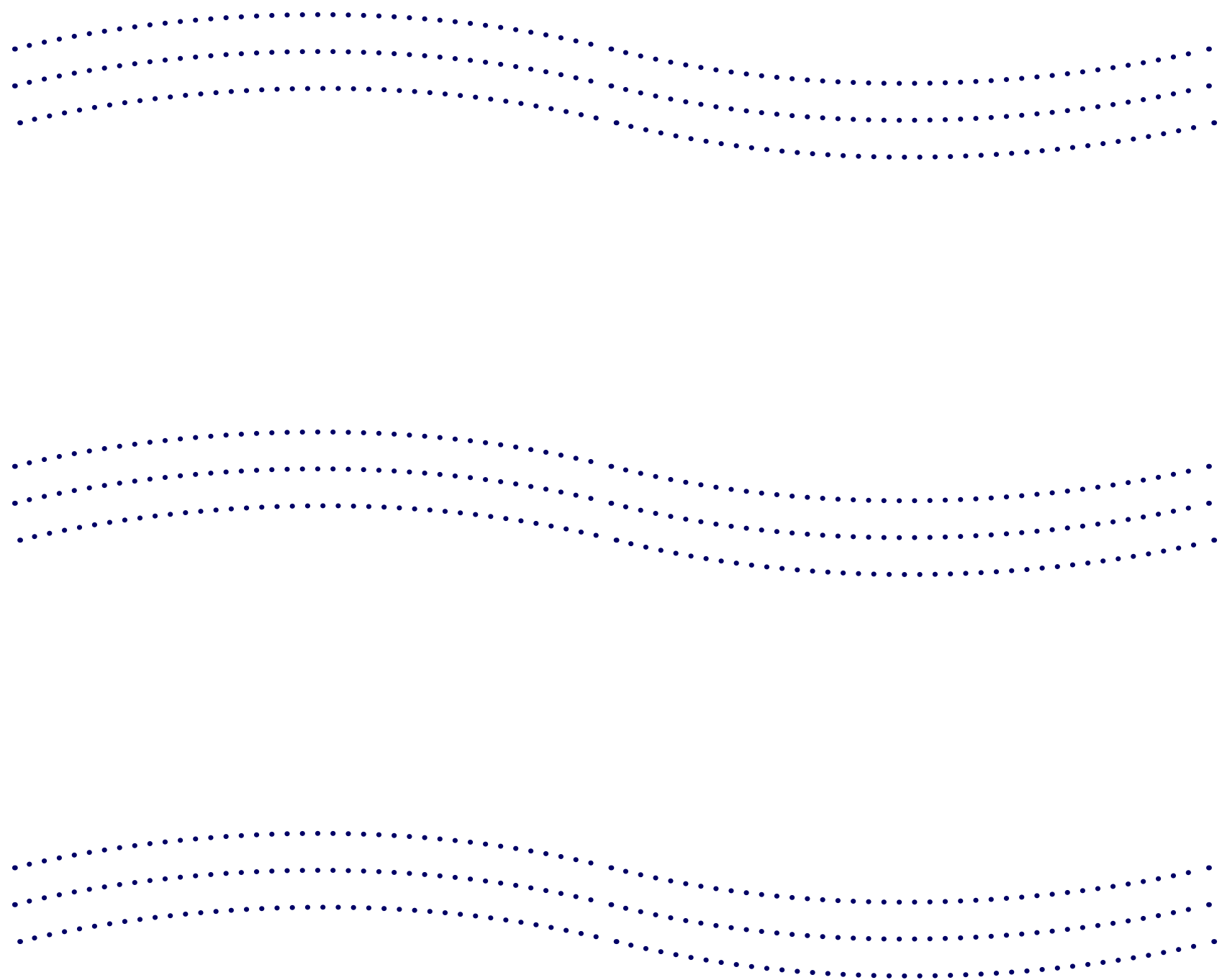